\title{
The Effect of the FIFA 11 + with Added Neck Exercises on Maximal Isometric Neck Strength and Peak Head Impact Magnitude During Heading: A Pilot Study
}

\author{
Kerry Peek ${ }^{1}$ (I) $\cdot$ Jordan Andersen $^{2} \cdot$ Marnee J. McKay $^{1} \cdot$ Theo Versteegh $^{3} \cdot$ Ian A. Gilchrist $^{4} \cdot$ Tim Meyer $^{5}$. \\ Andrew Gardner ${ }^{6,7}$ (i)
}

Accepted: 5 September 2021 / Published online: 29 September 2021

(c) The Author(s), under exclusive licence to Springer Nature Switzerland AG 2021

\begin{abstract}
Background Higher neck strength has been postulated to reduce head impact magnitude during purposeful heading in football.

Objectives This pilot trial explored the effect of a neck exercise programme on (1) neck strength and (2) head impact magnitude during heading in male and female adolescent football players.

Methods Boys and girls (aged 12-17 years) were randomised by team to the intervention (5 weeks of supervised neuromuscular neck exercises integrated into part 2 of the FIFA $11+$, completed three times per week) or the control group (usual part 2 of the FIFA $11+$, no neck exercises). Outcomes included isometric neck strength and head impact magnitude (peak linear acceleration and peak angular velocity) during standardised heading from a throw-in (at baseline and 6 weeks) plus completion of an evaluation survey by intervention players and coaches.

Results In total, 52 players ( $n=31$ intervention; $n=21$ control) completed the study. Mixed-model analysis of variance (ANOVA) revealed significant differences in neck strength variables $(p<0.001)$, peak linear acceleration $(p=0.04)$ and peak angular velocity $(p=0.04)$ between the intervention and control groups over time. Intervention players demonstrated increases in mean composite neck strength (53.8\% intervention vs $15.6 \%$ control) as well as decreases in mean peak linear head acceleration during heading $(-11.8 \%$ vs $-5.0 \%)$ from baseline to follow-up. Reduction in peak angular velocity was more pronounced in girls $(-27.7 \%)$ than boys $(-11.5 \%)$ in the intervention group. The addition of neck exercises into part 2 of the FIFA $11+$ was feasible and accepted by players and coaches.

Conclusion On average, players who completed neck exercises demonstrated an increase in isometric neck strength and a decrease in head impact magnitude during heading. These exercises were easily incorporated into usual training.

Australian New Zealand Clinical Trials Registry (no: ACTRN12619001375145).
\end{abstract}

\section{Introduction}

While heading has always been an integral part of football, there is growing uncertainty surrounding the role repeated head impacts from football heading has on brain health [1]. A recent cohort study reported that Scottish male professional football players $(n=7676)$ were more likely (hazard ratio 3.66 ; $95 \%$ confidence interval $[\mathrm{CI}] 2.88-4.65 ; p<0.001$ ) to be identified with a neurodegenerative disease when compared with matched controls $(n=23,028)$ from the general

Kerry Peek

Kerry.Peek@sydney.edu.au

Extended author information available on the last page of the article population [2]. Any impact to the head has the potential to cause a transmission of force to the brain [3]. Whether this results in detrimental stress and strain to brain tissue is likely related to head impact magnitude [3-6]. Head impact magnitude (including peak linear acceleration and angular velocity of the head) is influenced by many factors, including ball properties (mass and pressure) [7-9] and player characteristics (sex, age and neck strength) $[10,11]$.

Despite scientific uncertainty regarding the effects of purposeful heading [12-14], some football organisations have prohibited or restricted heading in young players $[15$, 16]. For example, the influential Football Associations of England, Scotland and Northern Ireland released updated heading guidance in 2020 and again in 2021, which state 


\section{Key Points}

Findings: Male and female adolescent players who completed a 5-week neuromuscular neck exercise programme integrated into part 2 of the FIFA $11+$ demonstrated an increase in isometric neck strength and a decrease in head impact magnitude during purposeful heading from a throw-in

Implications: Consideration should be given to adding neuromuscular neck exercises to part 2 of the FIFA $11+$ for adolescent football players to reduce head impact magnitude during heading

Caution: The effect of heading technique, particularly the differences in technique adopted by boys and girls, should be considered when evaluating the magnitude of head impact during purposeful heading in football

that heading should not be introduced in training sessions for players under 11 years, with restricted heading practice now including professional players [16]. No restrictions on heading in matches are evident in these guidelines, with heading practice recommended to commence with lighter balls [16]. Although prohibiting heading in training for young players will reduce the accumulative number of headers per season, it will not reduce age of first exposure if players can still head the ball in matches [17]. In addition, there are no sex distinctions evident in these guidelines despite boys physically growing at a faster rate than girls during puberty. Thus, a more inclusive preventative approach, such as improving the strength and neuromusculature control of the neck muscles, may offer a more holistic strategy to reduce head impact magnitude and heading burden in young players.

Greater neck strength has been postulated to attenuate resultant head kinematics during purposeful heading [18]. Stronger muscles have a greater cross-sectional area, tensile stiffness and ability to generate torque [18]. Newtonian physics have been used to compute head acceleration during purposeful headers for a wide variety of game situations, including the ability of the player to increase the effective mass of the head-and-neck segment during a heading manoeuvre [19]. Increasing the effective mass of the head-and-neck segment is achieved through isometrically or concentrically contracting selected neck musculature that stiffens the neck column and enhances the coupling of the head to the torso. The contracted neck muscles are then better able to absorb and dissipate the applied force on the head down through the neck to the body thereby minimising the resultant acceleration of the head on ball impact [19].
Child, adolescent and women football players are reportedly at greater risk of sustaining a concussion [20], with young athletes taking longer to recover than adult male players [21]. They also typically exhibit lower absolute neck strength, which may contribute to a higher risk of injury during heading [19]. Women collegiate football (i.e. soccer) players exhibited significantly lower neck strength and greater head accelerations during purposeful heading than men [22]. These sex-specific differences in neck strength have been attributed to reduced muscle mass and lower neck girth of women [10, 18, 23, 24]. Players with reduced neck strength often demonstrate an inability to generate the necessary stiffness of the neck column to control the head's response during purposeful heading [18].

A recent systematic review reported that injury reduction programmes that include neck exercises can reduce the risk of sport-related head and neck injuries, including concussion [25]. This review included two studies which explored the effect of the 'Activate' injury reduction programme (which includes neck exercises) designed for rugby union players $[26,27]$. The equivalent injury reduction programme in football is the FIFA $11+$ [28], which currently does not contain neck exercises. The FIFA $11+$ consists of three parts. Parts 1 and 3 contain running exercises, with part 2 consisting of strength, plyometric and balance exercises. The whole programme is recommended to be completed at every training session and takes approximately $20 \mathrm{~min}$ to complete (including 10 min to complete part 2) [28].

Despite evidence that higher neck strength is associated with lower head acceleration during heading [11, 29], very few studies have explored the effect of a neck exercise programme on head acceleration during heading in adolescent players [30], with none adding neck exercises to the current FIFA 11 + injury reduction programme. In addition, the feasibility and acceptability of a neck exercise programme is still to be explored.

\subsection{Objectives}

The primary objectives were to explore the effects of the FIFA $11+$ with added neck exercises on:

1. Isometric neck flexor, extensor and side flexor strength

2. Head impact magnitude during purposeful heading in male and female adolescent football players.

A secondary objective was to explore the adoption and feasibility of the programme. 


\section{Methods}

\subsection{Study Design and Setting}

This pilot trial was undertaken and reported in accordance with the Consolidated Standards of Reporting Trials (CONSORT) guidelines [31]. Approval for the research was granted by the University's Human Research Ethics Committee. This study was performed in accordance with the standards of ethics outlined in the Declaration of Helsinki and registered with the Australian New Zealand Clinical Trials Registry.

\subsection{Participants}

All players aged $12-17$ years $(n=88)$ who were registered with one high-level youth football club in New South Wales (NSW), Australia, were invited to participate. The club has boys' and girls' teams registered to play in the National Premier League in NSW, with players considered to be of a high-skill level for their age. Club players train three times per week for 40 weeks of the year, with a competitive playing season lasting approximately 22 weeks. Players were excluded if they self-reported symptoms or showed signs of a current head or neck injury during screening. Written consent was obtained from both the player and their parent/ legal guardian.

\subsection{Randomisation, Allocation Concealment and Blinding}

In accordance with coronavirus disease (COVID-19) pandemic restrictions, a limited number of research personnel were permitted at the club during data collection. This, in addition to the nature of the intervention, meant that blinding of the assessor and players was not possible. However, data from the respective devices (hand-held dynamometer and inertia measurement unit [IMU]) were extracted and analysed by a researcher blinded to group allocation. Teams, rather than players, were randomised to the intervention or control group. Players from eight teams (four boys' and four girls' teams) consented to participate. To limit large variations in player ages and sex allocated to each trial group, the club was split into junior (under-13 and under-14 boys' and girls' teams) and senior teams (under-15 boys' and girls' teams and under- 16 boys' team and under- 17 girls' team). A coin toss was then used to allocate one boys' and one girls' junior team to the intervention group (with the process repeated for the senior teams). This resulted in two boys' teams and two girls' teams being allocated to the intervention group (one junior and one senior team per sex) with the remaining four teams being allocated to the control group.

\subsection{Data Collection}

Data were collected between June and October 2020, with baseline assessment being conducted at the start of the resumption of training and competition following the easing of COVID-19 pandemic restrictions in community sport within NSW.

\subsubsection{Intervention}

The intervention consisted of players completing the FIFA $11+$ injury reduction programme with the addition of three neck exercises, three times per week for 5 weeks. The neck exercises were designed to be football-specific based on prior clinical application of neck exercises within a sporting environment. For the first exercise, based on the Versteegh Roll and Tuck (VRT), players were instructed to sit on the ground and hold their knees before rolling backwards and forwards as fast as they could whilst keeping their chin tucked in and not allowing their head to touch the ground/ floor (Fig. 1). Players completed the VRT for one set of the following (each lasting $30 \mathrm{~s}$ ): VRT with the head (1) pointed straight ahead, (2) turned to the left and (3) turned to the right. These exercises were integrated into part 2 of the FIFA $11+$ which all players at this club completed at the end of training (parts 1 and 3 were completed as pre-training warmup for all players). The VRT exercises were taught to players and coaching staff in the intervention arm by the project lead (KP) once all baseline measures had been collected. Club coaching staff supervised exercise completion at training, with the project lead attending a sample of four training sessions unannounced to review team and player adherence to the intervention. The VRT added less than 2 min to part 2 of the FIFA $11+$. Players were instructed to continue these exercises during the pre-season trial period as well as during the entire playing season.

\subsubsection{Control}

The control arm continued to complete the FIFA $11+$ as usual with part 2 at the end of training (which included strength, balance and plyometric exercises) $[28,32]$ but without the addition of neck exercises.

\subsection{Measures}

Screening The screening assessment included a questionnaire that collected demographic and sport experience information (e.g. age, sex, playing history, position, estimated number of headers per week [in training and match play]) in addition to previous head injury history (including concussion), as well as questions regarding any current head or neck pain or symptoms. Players then underwent a physical 
Fig. 1 Example of the Versteegh Roll and Tuck (VRT) neck flexor exercise for players in the intervention arm

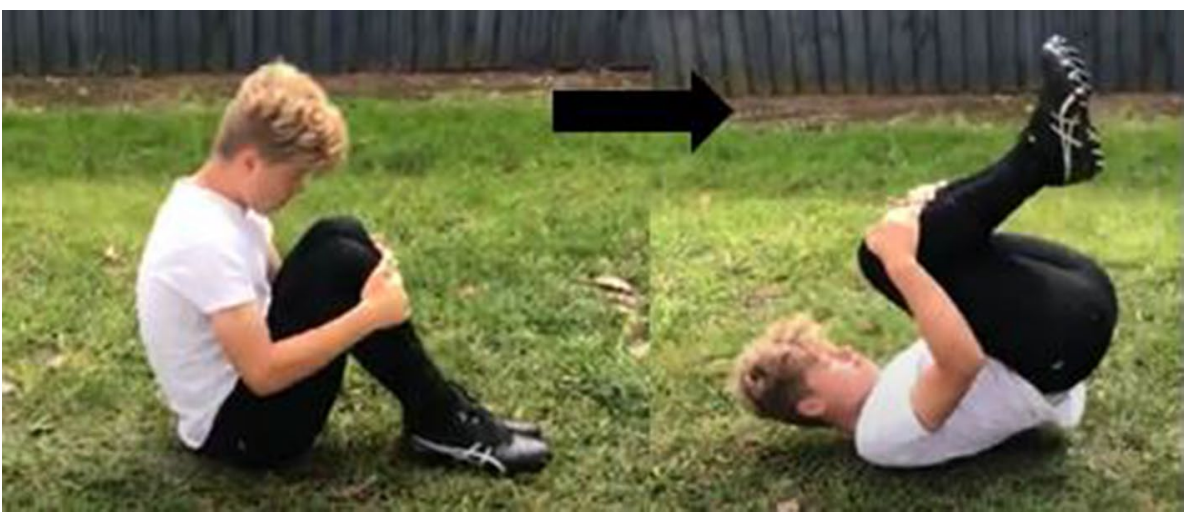

cervical spine assessment (including range of movement and palpation for posterior midline tenderness, which was defined as any player reporting tenderness on palpation greater than what may be typically expected when gently pressing on the skin over the back of the neck) to exclude any participants with current cervical spine issues.

Anthropometry Height (Seca $217^{\circledR}$, Germany) and weight (BF-522 Tanita ${ }^{\circledR}$, Japan) were collected with players in their standard training kit of light-weight shirt and shorts.

Muscle strength Maximal isometric muscle strength of the neck was defined as the maximal load in kilograms that could be applied to the player's head before deviation from a neutral testing position was observed. Assessment was undertaken using a previously reported 'break technique' protocol by the project lead using a Gatherer Systems ${ }^{\mathrm{TM}}$ hand-held dynamometer as used in earlier studies [33-35]. A head harness, with a load cell placed in series, was secured to the player's head. With the player's head in an anatomically neutral position, the same assessor applied manual incremental loading until a loss of test position occurred (i.e. to the point of head movement). The highest score from two repetitions was recorded. The test was repeated for each direction of movement (flexion, extension and left and right side flexion), with the same procedure and assessor measuring neck strength at baseline and follow-up [33, 34]. Prior to the assessment, players were advised of the assessment procedure and were given an opportunity to warm-up and practise. No adverse events were reported during muscle strength assessment. Figure 2 demonstrates the set-up for assessing maximal isometric muscle strength for the neck extensors.

Head impact magnitude All players completed heading trials using a size 5 Adidas ${ }^{\circledR}$ (Starlancer ${ }^{\mathrm{TM}}$ ) ball inflated to 5 pounds per square inch (psi).

Heading trials were supervised by the project lead using a standardised procedure described previously [9]. Players wore a close-fitting latex 'swim cap' that housed an IMU (AX6 ${ }^{\mathrm{TM}}$, Axivity ${ }^{\mathrm{TM}}$, Newcastle, UK) to measure head impact magnitude: linear acceleration (tri-axial accelerometer) and angular velocity (tri-axial gyroscope). The swim cap was placed on the player's head with the IMU positioned centrally at the base of the occiput, similar to the procedure used in earlier research [36]. Prior to data collection, we pilot tested our methods for capturing head impact magnitude during heading by mounting both this IMU (Axivity $^{\mathrm{TM}}$ ) and a hard-wired 25-g tri-axial accelerometer (Entran, EGA3-F-25 ${ }^{\mathrm{TM}}$ ) to participants' heads using the same swim cap method. The average error between the two devices was $0.07 \mathrm{~g}$, taken across 12 headers involving three participants.

A pro-licensed football coach (and ex-international player) delivered the balls from the sideline using an overhead throw-in using the following game scenario, familiar to the age group of players [37], which was provided to each player before the first throw-in: [9]

"Imagine that you are in a game, the ball has gone out of play and your team-mate is about to throw the ball to you from the side-line. You are going to head the ball back to your team-mate's feet so that they can play on."

For each heading trial, players were asked to stand within a $1 \times 1 \mathrm{~m}$ box positioned $5 \mathrm{~m}$ from the sideline on a standard outdoor football pitch. The validity assessment of headers was made subjectively by the same member of the research team for all headers at baseline and follow-up. For headers to be recorded as valid, players had to receive the ball on their forehead (as opposed to the top or side of the head), return the ball towards the coach delivering the throw-in, keep their feet grounded and be located inside the box when the header occurred. Otherwise, players could adopt any standing position or heading technique of their choosing. Invalid headers were repeated until at least two valid headers were recorded to a maximum of five headers to reduce heading burden. The intra-tester reliability of throw-ins was calculated from a sub-set of ten headers captured using slow motion video (120 frames per seconds) and using a process described previously [9]. 
Fig. 2 Dynamometry assessment position for neck extensors (image courtesy of Gatherer Systems)

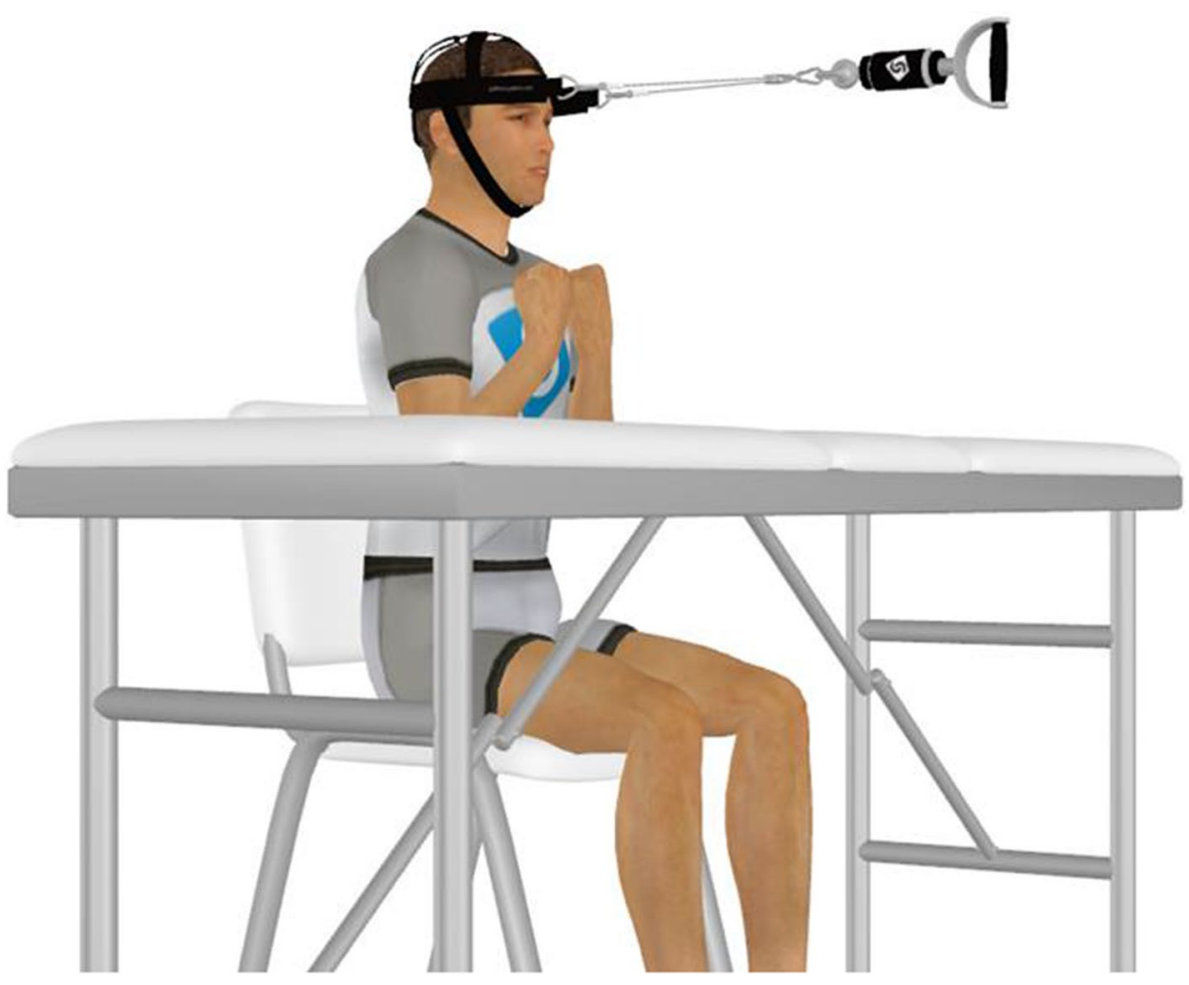

Data for linear acceleration (to a maximum of $16 \mathrm{~g}$ ) and angular velocity (to a maximum of $1000^{\circ} / \mathrm{s}$ ) were sampled along each orthogonal axis (i.e. $x, y, z$ ) at $800 \mathrm{~Hz}$. Time series data were downloaded to the OmGui software (Axivity $^{\mathrm{TM}}$, Newcastle, UK) as continuous wave accelerometer (*.cwa) files, which were converted into *.csv files. Resultant linear acceleration and angular velocity were calculated from the raw data using the Pythagorean equation $\left(r=\sqrt{x^{2}+y^{2}+z^{2}}\right)$, and peak values were extracted from each heading trial to represent head impact magnitude [9] in MATLAB (R2018b, Mathworks Inc. ${ }^{\mathrm{TM}}$ ). As well as visually assessing whether each header was valid, the time each header occurred in the field during data collection was manually recorded (in hours:minutes:seconds). The time stamp of the data in the Axivity file (also recorded in hours:minutes:seconds) was then compared to the manual time recorded for each header. Figure 3 shows a typical time series of accelerometer data for two heading trials (see also Supplementary Appendix in the electronic supplementary material).

Isometric neck strength and IMU data were collected using the same standardised procedure at baseline and 6 weeks, following the 5-week intervention period.

Evaluation questionnaire The feasibility and acceptability of the trial's measures and intervention programme were evaluated using an anonymous questionnaire handed out to players at the end of the season. The questionnaire consisted of a number of statements, with players asked whether they agreed or disagreed with each statement based on a 5-point
Likert scale $(1=$ completely disagree; $5=$ completely agree $)$. The coaches involved in supervising the neck exercise intervention were also requested to complete a similar questionnaire (see Supplementary Appendix In the electronic supplementary material).

\subsection{Sample Size}

An a priori sample size calculation was undertaken using G*Power 2019 (version 3.1.9.7, Dusseldorf, Germany). Using a mixed-model analysis of variance (ANOVA) with within-between group interaction $(f>0.25$, power 0.90 , $\alpha=0.05$ ), a minimum group size of 46 participants was

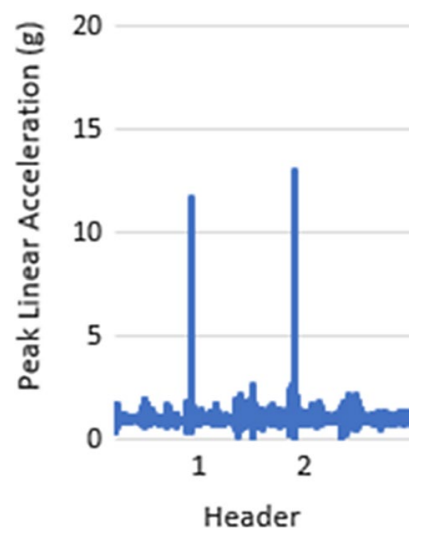

Fig. 3 Example of one player's peak linear acceleration for 2 headers 
required, which was increased to 50 to account for a potential $10 \%$ dropout rate.

\subsubsection{Statistical Methods}

Analyses were performed using Stata (College Station, Texas, USA). The level of significance set a priori was $\alpha=0.05$.

Descriptive data for participant demographics and the evaluation questionnaire are presented using counts, means and standard deviations. Baseline differences in group characteristic measures were analysed by $t$ tests for continuous variables. Intra-tester reliability of the throw-ins as well as neck strength assessment were calculated using intra-class correlation coefficients (ICCs) (two-way mixed effects model using absolute agreement), with magnitudes interpreted as poor (less than 0.50 ), moderate $(0.51-0.75)$, good (0.75-0.90) and excellent (more than 0.91) [38].

Neck strength variables are presented per direction of movement (flexion, extension, left and right side flexion) as well as a composite score (the sum of strength values for the four directions of movement divided by four). In addition, as participants were adolescent players at various stages of growth, each player's composite neck strength score was adjusted to their body mass index (BMI) [39]. BMI was calculated using the formula $\mathrm{BMI}=\mathrm{kg} / \mathrm{m}^{2}$, where the player's mass in kilograms was divided by their height in metres squared.

For all outcome variables the minimal detectable change (MDC) was calculated. The MDC is the minimal amount of within-subject variability that a measurement can show that is not due to measurement error or chance [40]. The standard error of measurement (SEM) was used to calculate the MDC using the following formula: $\mathrm{MDC}=\mathrm{SEM} \times 1.96 \times \sqrt{ } 2[40]$.

Comparative analyses between groups were performed using an intention-to-treat approach, where every player who was randomised to either the intervention or control group was included in the analysis. Intention-to-treat analyses can assist in avoiding overoptimistic estimates of the efficacy of an intervention that result from the removal of non-adherers by accepting that non-adherence and protocol deviations are likely to occur in real life [41]. To analyse pre and post effects and group differences, the mixed-model ANOVA was applied with time (baseline and follow-up) as a within-player factor and group as a between-player factor. Separate mixed-model ANOVAs were performed with neck strength (in flexion, extension, left and right side flexion and composite neck strength) as well as peak linear head acceleration and peak angular velocity as the dependent variable. With each of these ANOVAs, the hypothesis of interest was the two-way interaction of group*time. Before completing the mixed-model ANOVA, the normality of data distribution $(p \geq 0.05)$ of each dependent variable in each combination was confirmed using the Shapiro-Wilk test, with homogeneity of variance $(p \geq 0.05)$ tested with Levene's test. Calculation of effect size using partial eta squared (with 95\% CI) was performed, with interpretations of no effect $(<0.01)$, small (0.02-0.05) medium (0.06-0.13) and large ( $>0.14)$. Means (SD), $f$ values and $p$ values are reported.

\section{Results}

From a total number of 88 invited players, 59 players (67\%) consented to participate. One player was excluded due to sustaining a recent concussion whilst playing school sport (badminton), leading to 58 players from eight teams being randomised into the intervention or control arm of the trial (Fig. 4). No other players were excluded following the screening assessment. Six players did not complete the baseline heading trials and were removed from the analysis. As players were randomised via their teams, this led to an unequal number of players in each group (intervention $n=31$; control $n=21$ ).

Table 1 reports the mean baseline characteristics of players. For combined boys' and girls' data, $t$ tests revealed differences in baseline characteristics for hours trained per week and number of self-reported headers per week, with players in the control group completing more hours of training and more self-reported headers than players in the intervention group. There were no significant differences between groups for any of the girls' baseline characteristics, but significant between-group differences were observed for the boys, including age, height and weight, with players in the control group tending to be older, heavier and taller.

The ICC for throw-ins was calculated at $0.78(95 \% \mathrm{CI}$ 0.71-0.82), demonstrating good reliability. The ICC of neck strength assessment demonstrated good (flexion 0.80, right side flexion 0.84 and left side flexion 0.82 ) to excellent reliability (extension 0.92 ), similar to values reported in earlier studies $[42,43]$.

\subsection{Intervention Effects on Neck Strength Variables and Head Impact Magnitude}

Although within-group and between-group $(p \geq 0.05)$ sex differences over time were not significant, we chose to perform separate mixed-model ANOVAs for boys' and girls' data as well as the combined data. This was done to further explore whether any sex differences in results were apparent given the variation in heading technique subjectively noted between the boys and girls during the heading trials (Table 2). The difference between composite neck strength and composite neck strength adjusted to BMI was nonsignificant $(p \geq 0.05)$; therefore, only unadjusted scores are reported (Table 2). 


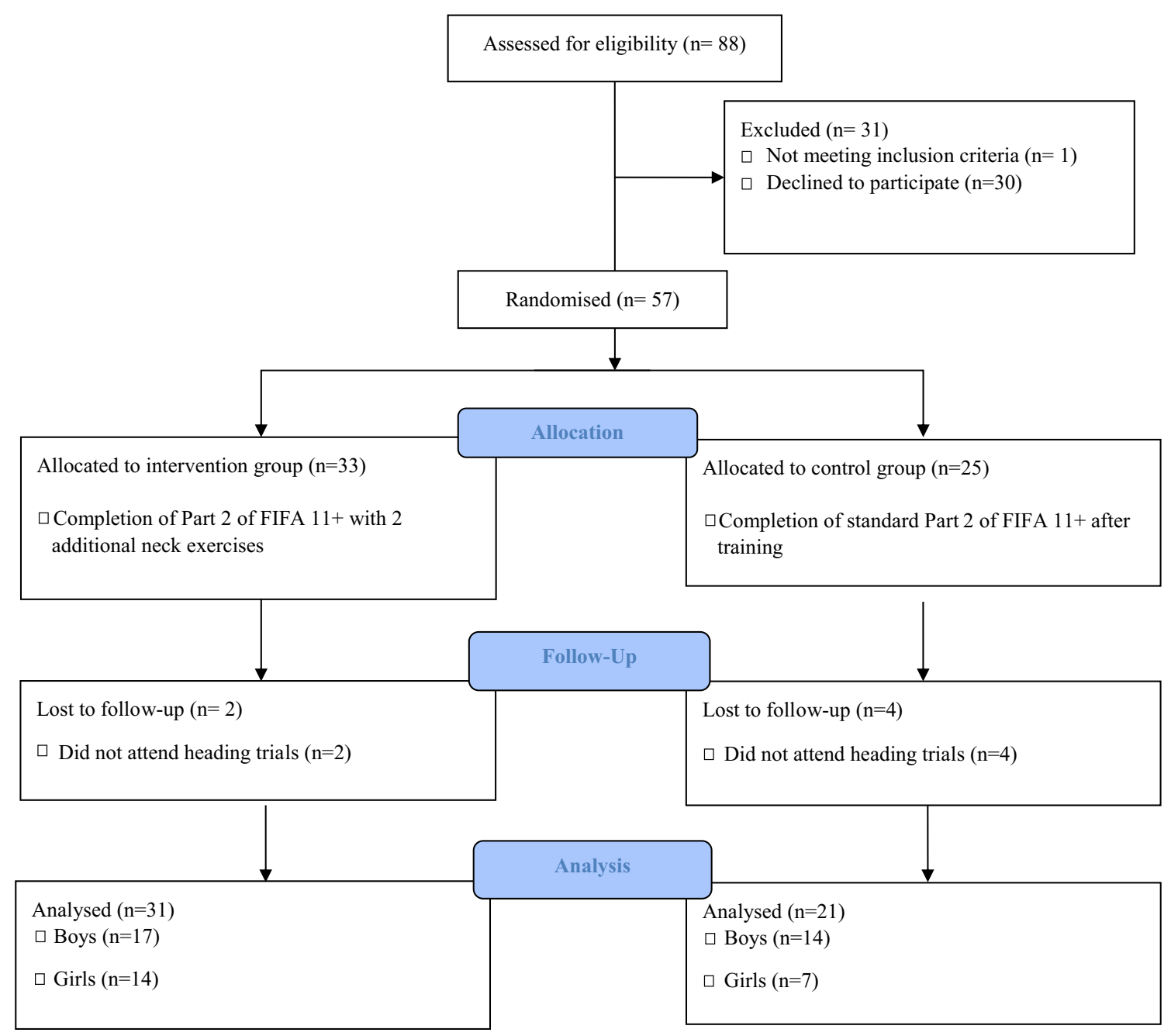

Fig. 4 CONSORT flow diagram of study protocol. CONSORT Consolidated Standards of Reporting Trials

For players in the intervention group, the change scores from pre to post test for all variables exceeded the MDC by more than $10 \%$ except for peak angular velocity, which exceeded the MDC by less than 10\%. Mixed-model ANOVAs for the combined and girls' data revealed large effects for neck strength variables $\left(\eta^{2}>0.22 ; p<0.001\right)$, medium effects for peak linear acceleration $\left(\eta^{2}>0.08 ; p<0.05\right)$ and medium effects for peak angular velocity $\left(\eta^{2}>0.07\right.$; $p<0.05)$ between the intervention and control groups over time. Boys' data demonstrated large effects for neck strength variables $\left(\eta^{2}>0.28 ; p \leq 0.001\right)$, medium effects for peak linear acceleration $\left(\eta^{2} 0.13 ; p=0.05\right)$ and small effects for angular velocity $\left(\eta^{2} 0.03 ; p=0.13\right)$, although neither peak linear acceleration nor peak angular velocity were statistically significant due to the lower 95\% CI crossing zero. Intervention players demonstrated greater increases in mean composite neck strength (53.8\% intervention vs $15.6 \%$ control) as well as greater decreases in mean peak linear head acceleration during heading $(-11.8 \%$ vs $-5.0 \%)$ from baseline to follow-up when compared with control players. Reduction in peak angular velocity was more pronounced in girls $(-27.7 \%)$ than in boys $(-11.5 \%)$ in the intervention group.

\subsection{Acceptability and Feasibility of the Programme}

The mean results for the player evaluation survey to assess the acceptability of the trial process can be seen in Table 3 (statements 1-5). All players reported a positive experience with neck strength testing ( $>3.57$ ), with no issues reported with neck strength testing $(>4.50)$ or heading trials $(>4.41)$. Players in both groups also reported feeling more confident heading a ball following involvement in the study ( $>3.43)$.

Players involved in the intervention arm were given five additional statements (Table 3; statements 6-10) to assess the acceptability and feasibility of the neck exercise 
Table 1 Baseline characteristics (mean [SD] for age, height, weight, body mass index, playing experience, training volume and self-reported number of headers per week for males and females and combined data

\begin{tabular}{|c|c|c|c|c|c|c|}
\hline \multirow[t]{2}{*}{ Characteristic } & \multicolumn{3}{|c|}{ Intervention, mean (SD) } & \multicolumn{3}{|l|}{ Control, mean (SD) } \\
\hline & Combined $(n=31)$ & Boys $(n=17)$ & Girls $(n=14)$ & Combined $(n=21)$ & Boys $(n=14)$ & Girls $(n=7)$ \\
\hline Age (years) & $14.35(0.29)$ & $13.80 *(0.24)$ & $15.00(0.55)$ & $14.95(0.21)$ & $15.28 *(0.26)$ & $14.29(1.88)$ \\
\hline Height (metres) & $1.64(0.01)$ & $1.64 *(0.02)$ & $1.63(0.02)$ & $1.69(0.02)$ & $1.73 *(0.02)$ & $1.59(0.03)$ \\
\hline Weight (kilograms) & $53.06(1.69)$ & $51.52 *(2.17)$ & $54.93(2.66)$ & $55.90(2.25)$ & $60.14 *(2.40)$ & $47.42(2.83)$ \\
\hline Body mass index & $19.63(0.41)$ & $19.01(0.46)$ & $20.40(0.69)$ & $19.39(0.37)$ & $19.84(0.44)$ & $18.48(0.59)$ \\
\hline Years played & $8.51(0.40)$ & $8.41(0.51)$ & $8.64(0.65)$ & $9.67(0.34)$ & $9.57(0.48)$ & $9.87(0.34)$ \\
\hline Hours training per week & $4.83 *(0.27)$ & $4.70 *(0.28)$ & $5.00(0.50)$ & $6.21 *(0.56)$ & $6.78 *(0.79)$ & $5.07(0.38)$ \\
\hline Headers per week $(n)$ & $5.80 *(0.97)$ & $5.94 *(1.22)$ & $5.64(1.60)$ & $13.00 *(2.71)$ & $16.92 *(3.60)$ & $5.14(1.40)$ \\
\hline
\end{tabular}

$S D$ standard deviation

*Difference in baseline characteristics $p<0.05$

intervention. Intervention players all agreed or completely agreed that completing neck exercises was a positive experience ( $>4.21)$, the exercises were easy to complete $(>4.44)$, did not take too long $(>4.42)$, provided personal benefit $(>4.05)$ as well as team benefit $(>4.00)$.

Coaches $(n=4)$ involved in supervising the intervention agreed or completely agreed that the exercise programme was easy to schedule (mean 4.50 [SD 0.57]), did not take too long to complete (4.74 [SD 0.50]), benefitted the players (4.50 [SD 0.57]) and club (5.00 [SD 0.00]) and that intervention players appeared more confident heading a ball following the intervention (4.50 [SD 0.57]).

\section{Discussion}

This is one of the few studies to have explored the effects of a neck exercise programme on head impact magnitude during heading in adolescent male and female football players. Despite adding less than 2 min to part 2 of the FIFA $11+$, boys and girls in the intervention group were able to demonstrate large increases in neck strength in only 5 weeks (Table 2). In addition, players in the intervention group demonstrated a medium effect in reducing peak linear head acceleration $\left(\eta^{2}=0.08 ; 95 \%\right.$ CI $\left.0.01-0.17\right)$ and a medium effect in reducing peak angular velocity $\left(\eta^{2}=0.07 ; 95 \%\right.$ CI 0.01-0.15) after the 5-week training programme. This effect was higher in girls than boys, which is possibly unsurprising given that girls in the intervention group generally exhibited weaker neck strength than boys at baseline, with slightly greater improvements in neck strength over the intervention period. Our results also demonstrated that adding neck exercises to part 2 of the FIFA 11 + was accepted by the players and coaches in this study.

An earlier study employing a twice weekly neck exercise programme for 14 weeks in 27 football players aged $15-18$ years ( $n=14$ in the intervention group) [30] reported a reduction in peak linear acceleration of the head during heading for players involved in the intervention group $\left(\eta^{2}>0.21\right)$. This intervention effect was greater than the one seen in our study $\left(\eta^{2}=0.08\right)$, possibly related to the longer intervention period (which was almost three times longer). In contrast, a 6-week strength training programme of the neck flexors and extensors in 33 male football players did not report a significant change in neck strength and little change in head impact magnitude during heading [44]. The difference in findings may be explained by the variations in study methodology and training intervention. In our study and the study by Müller and colleagues [30], the neck exercises predominantly emphasised neuromuscular control rather than muscle strengthening alone, plus the balls delivered in these studies replicated an accepted game scenario. A throw-in was used in our study, while the study by Müller and colleagues used a ball pitching machine to replicate a kicked ball delivery [30]. In contrast, the study by Becker and colleagues used strength based exercises with resistance bands or static resistance provided by another player, with the players asked to head a stationary pendular ball attached to the ceiling of the laboratory [44].

The notion that greater neck strength can reduce head acceleration during heading (and potentially reduce injury risk) is based on the premise that stronger, stiffer necks increase coupling of the head to the body and thus help stabilise the head on ball impact [19]. However, although absolute neck strength is an important factor, heading in football is a fast, dynamic action, placing importance not just on muscle strength but on the ability of players to recruit these muscles very quickly. Exercises that aim to enhance the neuromuscular responsiveness of the neck muscles are designed to help players generate the maximal amount of tension in the shortest amount of time. Thus, increasing the coupling of the head-neck-torso enables players to resist head acceleration on ball impact. Peak linear acceleration was shown to decrease in players who pre-tensed their neck 
Table 2 Intervention effects of mean maximal isometric neck strength variables, mean peak linear head acceleration and mean peak angular velocity between intervention and control group from baseline to 5-week re-test for combined, male and female data

\begin{tabular}{|c|c|c|c|c|c|c|}
\hline Combined boys and girls $(n=52)$ & $\begin{array}{l}\text { Minimal } \\
\text { detectable } \\
\text { change } \\
\text { Pre; post }\end{array}$ & $\begin{array}{l}\text { Intervention }(n=31) \\
\text { Pre; post scores } \\
\text { Change score }(\mathrm{SD}) \\
\%\end{array}$ & $\begin{array}{l}\text { Control }(n=21) \\
\text { Pre; post scores } \\
\text { Change score }(\mathrm{SD}) \\
\%\end{array}$ & $F(1,102)$ & $p$ value & Effect size $\eta^{2}(95 \% \mathrm{CI})$ \\
\hline Extensors (kg) & $2.56 \uparrow$ & $\begin{array}{l}13.67 ; 20.89 \\
7.21(5.14) \\
52.8 \%\end{array}$ & $\begin{array}{l}21.54 ; 23.54 \\
2.00(3.58) \\
9.3 \%\end{array}$ & 9.54 & $<0.001$ & $0.22(0.08-0.34)^{* *}$ \\
\hline Flexors (kg) & $1.39 \uparrow$ & $\begin{array}{l}8.44 ; 13.16 \\
4.71(2.79) \\
55.9 \%\end{array}$ & $\begin{array}{l}13.66 ; 14.81 \\
1.15(2.84) \\
8.5 \%\end{array}$ & 10.99 & $<0.001$ & $0.25(0.10-0.36)^{* *}$ \\
\hline Right side flexors (kg) & $1.63 \uparrow$ & $\begin{array}{l}8.74 ; 13.70 \\
4.96(3.26) \\
56.8 \%\end{array}$ & $\begin{array}{l}12.67 ; 16.15 \\
3.48(3.04) \\
8.4 \%\end{array}$ & 12.29 & $<0.001$ & $0.27(0.12-0.38)^{* *}$ \\
\hline Left side flexors (kg) & $1.65 \uparrow$ & $\begin{array}{l}9.02 ; 13.61 \\
4.59(3.30) \\
50.9 \%\end{array}$ & $\begin{array}{l}13.37 ; 16.42 \\
3.05(3.52) \\
22.8 \%\end{array}$ & 11.62 & $<0.001$ & $0.26(0.11-0.37)^{* *}$ \\
\hline Composite neck strength $(\mathrm{kg})$ & $1.67 \uparrow$ & $\begin{array}{l}10.37 ; 15.95 \\
5.58(3.34) \\
53.8 \%\end{array}$ & $\begin{array}{l}15.94 ; 18.43 \\
2.49(3.97) \\
15.6 \%\end{array}$ & 12.47 & $<0.001$ & $0.27(0.12-0.39)^{* *}$ \\
\hline Peak linear acceleration (g) & $-1.30 \uparrow$ & $\begin{array}{l}12.85 ; 11.34 \\
-1.51(1.62) \\
-11.8 \%\end{array}$ & $\begin{array}{l}11.53 ; 10.95 \\
-0.58(2.83) \\
-5.0 \%\end{array}$ & 2.77 & 0.04 & $0.08(0.01-0.17)^{*}$ \\
\hline Peak angular velocity (dps) & $-79.10 \leftrightarrow$ & $\begin{array}{l}497.09 ; 415.31 \\
-81.78(202.76) \\
-16.5 \%\end{array}$ & $\begin{array}{l}399.09 ; 339.41 \\
-59.68(311.98) \\
-15.0 \%\end{array}$ & 2.68 & 0.04 & $0.07(0.01-0.15)^{*}$ \\
\hline Boys $(n=31)$ & $\begin{array}{l}\text { Minimal } \\
\text { detectable } \\
\text { change } \\
\text { Pre; post }\end{array}$ & $\begin{array}{l}\text { Intervention }(n=17) \\
\text { Pre; post scores } \\
\text { Change score (SD) } \\
\%\end{array}$ & $\begin{array}{l}\text { Control }(n=14) \\
\text { Pre; post scores } \\
\text { Change score (SD) } \\
\%\end{array}$ & $F(1,60)$ & $p$ value & Effect size $\eta^{2}(95 \% \mathrm{CI})$ \\
\hline Extensors (kg) & $3.88 \uparrow$ & $\begin{array}{l}14.64 ; 22.07 \\
7.44(5.77) \\
50.8 \%\end{array}$ & $\begin{array}{l}25.31 ; 26.89 \\
1.58(3.18) \\
6.2 \%\end{array}$ & 10.14 & $<0.001$ & $0.34(0.14-0.48)^{* *}$ \\
\hline Flexors (kg) & $2.19 \uparrow$ & $\begin{array}{l}9.26 ; 14.23 \\
4.96(3.25) \\
53.7 \%\end{array}$ & $\begin{array}{l}16.17 ; 16.54 \\
0.37(2.49) \\
2.3 \%\end{array}$ & 7.45 & $<0.001$ & $0.28(0.08-0.41)^{* *}$ \\
\hline Right side flexors (kg) & $2.51 \uparrow$ & $\begin{array}{l}9.78 ; 15.28 \\
5.50(3.73) \\
56.2 \%\end{array}$ & $\begin{array}{l}14.56 ; 18.35 \\
3.79(3.29) \\
26.0 \%\end{array}$ & 9.93 & $<0.001$ & $0.34(0.31-0.65)^{* *}$ \\
\hline Left side flexors $(\mathrm{kg})$ & $2.41 \uparrow$ & $\begin{array}{l}9.87 ; 15.00 \\
5.13(3.58) \\
52.0 \%\end{array}$ & $\begin{array}{l}15.50 ; 18.53 \\
3.03(3.58) \\
19.5 \%\end{array}$ & 9.35 & $<0.001$ & $0.33(0.13-0.47)^{* *}$ \\
\hline Composite neck strength $(\mathrm{kg})$ & $2.53 \uparrow$ & $\begin{array}{l}11.32 ; 17.32 \\
6.00(3.76) \\
53.0 \%\end{array}$ & $\begin{array}{l}18.60 ; 20.86 \\
2.26(4.15) \\
12.2 \%\end{array}$ & 10.62 & $<0.001$ & $0.35(0.14-0.49)^{* *}$ \\
\hline Peak linear acceleration (g) & $-0.71 \uparrow$ & $\begin{array}{l}13.74 ; 12.84 \\
-0.89(2.95) \\
-6.6 \%\end{array}$ & $\begin{array}{l}11.69 ; 11.21 \\
-0.48(2.87) \\
-4.1 \%\end{array}$ & 2.42 & 0.05 & $0.13(0.00-0.28)^{*}$ \\
\hline Peak angular velocity (dps) & $-71.67 \leftrightarrow$ & $\begin{array}{l}629.14 ; 556.83 \\
-72.31(103.78) \\
-11.5 \%\end{array}$ & $\begin{array}{l}441.18 ; 395.14 \\
-45.85(281.67) \\
-10.5 \%\end{array}$ & 1.05 & 0.13 & $0.03(0.00-0.09)$ \\
\hline
\end{tabular}


Table 2 (continued)

\begin{tabular}{|c|c|c|c|c|c|c|}
\hline Girls $(n=21)$ & $\begin{array}{l}\text { Minimal } \\
\text { detectable } \\
\text { change } \\
\text { Pre; post }\end{array}$ & $\begin{array}{l}\text { Intervention }(n=14) \\
\text { Pre; post scores } \\
\text { Change score }(\mathrm{SD}) \\
\%\end{array}$ & $\begin{array}{l}\text { Control }(n=7) \\
\text { Pre; post scores } \\
\text { Change score }(\mathrm{SD}) \\
\%\end{array}$ & $F(1,40)$ & $p$ value & Effect size $\eta^{2}(95 \% \mathrm{CI})$ \\
\hline Extensors (kg) & $3.03 \uparrow$ & $\begin{array}{l}12.52 ; 19.45 \\
6.93(4.46) \\
55.4 \%\end{array}$ & $\begin{array}{l}14.00 ; 16.86 \\
2.86(3.98) \\
20.4 \%\end{array}$ & 5.61 & 0.003 & $0.30(0.05-0.46)^{* *}$ \\
\hline Flexors (kg) & $1.62 \uparrow$ & $\begin{array}{l}7.44 ; 11.86 \\
4.41(2.19) \\
59.4 \%\end{array}$ & $\begin{array}{l}8.68 ; 11.35 \\
2.67(3.19) \\
30.8 \%\end{array}$ & 4.84 & 0.006 & $0.28(0.03-0.44)^{* *}$ \\
\hline Right side flexors (kg) & $1.90 \uparrow$ & $\begin{array}{l}7.48 ; 11.77 \\
4.29(2.51) \\
57.4 \%\end{array}$ & $\begin{array}{l}8.89 ; 11.76 \\
2.87(2.79) \\
32.3 \%\end{array}$ & 4.84 & 0.006 & $0.28(0.03-0.44)^{* *}$ \\
\hline Left side flexors $(\mathrm{kg})$ & $2.16 \uparrow$ & $\begin{array}{l}7.98 ; 11.91 \\
3.92(2.91) \\
49.2 \%\end{array}$ & $\begin{array}{l}9.10 ; 12.20 \\
3.10(3.48) \\
34.1 \%\end{array}$ & 4.66 & 0.007 & $0.33(0.11-0.46)^{* *}$ \\
\hline Composite neck strength (kg) & $2.07 \uparrow$ & $\begin{array}{l}9.21 ; 14.28 \\
5.07(2.80) \\
55.0 \%\end{array}$ & $\begin{array}{l}10.60 ; 13.60 \\
3.00(2.89) \\
28.3 \%\end{array}$ & 5.81 & 0.002 & $0.31(0.06-0.47)^{* *}$ \\
\hline Peak linear acceleration $(\mathrm{g})$ & $-1.54 \uparrow$ & $\begin{array}{l}11.78 ; 9.75 \\
-2.03(2.08) \\
-17.2 \%\end{array}$ & $\begin{array}{l}11.21 ; 10.44 \\
-0.77(2.80) \\
-6.9 \%\end{array}$ & 3.25 & 0.03 & $0.14(0.01-0.28)^{* *}$ \\
\hline Peak angular velocity (dps) & $-91.87 \leftrightarrow$ & $\begin{array}{l}336.75 ; 243.48 \\
-93.27(210.43) \\
-27.7 \%\end{array}$ & $\begin{array}{l}314.91 ; 330.16 \\
15.25(314.49) \\
4.8 \%\end{array}$ & 5.34 & 0.002 & $0.21(0.03-0.36)^{* *}$ \\
\hline
\end{tabular}

Effect size: * medium and $* *$ large effect

$C I$ confidence interval, $d p s$ degrees per second, $S D$ standard deviation, $\uparrow$ exceeds minimal detectable change by $>10 \%$, $\leftrightarrow$ exceeds minimal detectable change by $<10 \%$

Table 3 Player-reported evaluation results, mean (SD) (scored from $1=$ completely disagree to $5=$ completely agree)

\begin{tabular}{|c|c|c|c|c|c|c|}
\hline \multirow[t]{2}{*}{ Statement } & \multicolumn{3}{|c|}{ Intervention, mean (SD) } & \multicolumn{3}{|l|}{ Control, mean (SD) } \\
\hline & Combined $(n=31)$ & Boys $(n=17)$ & Girls $(n=14)$ & Combined $(n=21)$ & Boys $(n=14)$ & Girls $(n=7)$ \\
\hline $\begin{array}{l}\text { 1. Being involved with neck strength test- } \\
\text { ing was a positive experience for me }\end{array}$ & $4.21(0.09)$ & $4.04(0.14)$ & $4.39(0.11)$ & $3.78(0.80)$ & $3.57(0.75)$ & $4.00(0.78)$ \\
\hline $\begin{array}{l}\text { 2. The research team answered any ques- } \\
\text { tions or concerns that I had }\end{array}$ & $4.24(0.13)$ & $4.26(0.18)$ & $4.22(0.20)$ & $4.29(0.72)$ & $3.85(0.36)$ & $4.71(0.73)$ \\
\hline $\begin{array}{l}\text { 3. I did not have any issues with the neck } \\
\text { strength testing }\end{array}$ & $4.54(0.11)$ & $4.57(0.15)$ & $4.50(0.12)$ & $4.78(0.42)$ & $4.71(0.46)$ & $4.85(0.36)$ \\
\hline $\begin{array}{l}\text { 4. I did not have any issues with the head- } \\
\text { ing trials }\end{array}$ & $4.41(0.11)$ & $4.52(0.15)$ & $4.27(0.15)$ & $4.71(0.46)$ & $4.71(0.46)$ & $4.71(0.46)$ \\
\hline $\begin{array}{l}\text { 5. I feel more confident heading a ball } \\
\text { after being involved in this study }\end{array}$ & $4.00(0.13)$ & $4.21(0.20$ & $3.78(0.17)$ & $3.5(1.01)$ & $3.57(10.08)$ & $3.43(0.93)$ \\
\hline $\begin{array}{l}\text { 6. Being involved with neck strengthen- } \\
\text { ing exercises was a positive experience } \\
\text { for me }\end{array}$ & $4.27(0.09)$ & $4.21(0.14)$ & $4.33(0.11)$ & & & \\
\hline $\begin{array}{l}\text { 7. The neck exercise programme was easy } \\
\text { to complete }\end{array}$ & $4.48(0.09)$ & $4.52(0.14)$ & $4.44(0.14)$ & & & \\
\hline $\begin{array}{l}\text { 8. The neck exercise programme did not } \\
\text { take too long for me to complete }\end{array}$ & $4.45(0.13)$ & $4.42(0.15)$ & $4.5(0.12)$ & & & \\
\hline $\begin{array}{l}\text { 9. I feel that I benefitted from neck exer- } \\
\text { cises }\end{array}$ & $4.16(0.13)$ & $4.26(0.20)$ & $4.05(0.17)$ & & & \\
\hline $\begin{array}{l}\text { 10. I feel our team benefitted from com- } \\
\text { pleting neck exercises }\end{array}$ & $4.14(0.12)$ & $4.26(0.18)$ & $4.00(0.18)$ & & & \\
\hline
\end{tabular}

SD standard deviation 
muscles compared with players who did not (relaxed condition) for low ball velocities $(6 \mathrm{~m} / \mathrm{s})$ during a straight, standing header in an earlier study [45]. This result, although less pronounced, was replicated at higher ball velocities $(8 \mathrm{~m} / \mathrm{s})$. During the pre-tensed condition, the right and left sternocleidomastoid muscles were active for a longer period of time when compared to the relaxed condition [45]. The smaller difference at the high ball velocity may indicate that at higher velocities, players need to recruit their muscles over a much shorter period of time. To this end, implementation of training stimuli specifically designed to enhance the neuromuscular responsiveness (e.g. rate of force development) of the neck musculature may be the most appropriate training method to minimise head acceleration during heading in football players. The neck exercises used in our study were deliberately chosen to focus more on neuromuscular control of the head rather than pure strength of the neck muscles.

One surprising finding in our study was that girls generally demonstrated lower peak linear acceleration and peak angular velocity when compared with boys despite generally having weaker isometric neck strength. This finding is inconsistent with findings from previous studies [11, 30, 46]. We suspect this was a result of the differences in standing position and heading technique observed between boys and girls. While the heading technique was not standardised, it was noted by both the pro-licence coach and project lead that boys tended to adopt a staggered lunge stance and were more actively engaged in their heading technique, leading to a shift in weight from their back to their front foot to meet and 'attack the ball'. In contrast, girls tended to stand with their feet shoulder-width apart and were more passive in their approach by waiting for the ball to reach them. Whether the boys and girls in our study were coached to head a ball differently or had varying levels of heading experience cannot be extrapolated from these results, although, boys did self-report more headers per week than girls, possibly indicating more opportunities to practise and develop heading technique proficiency. This interpretation is supported by previous research that more experienced players adopt different heading technique to less experienced players, with novices and amateur players demonstrating the largest variation in head impact magnitudes during heading when compared with elite players [36]. It was also reported in a study of 34 adult athletes ( $n=20$ men; $n=14$ women) that despite women having lower neck muscle strength than men, women demonstrated increased activation of the sternocleidomastoid muscle to stabilise the head during laboratory-based head perturbations [47]. This finding possibly suggests that men and women use different strategies to stabilise the head during heading. Further, boys in our study tended to be more accurate in returning the ball to the feet of the coach delivering the throw-in (as instructed) than girls, who appeared to have less control of the ball (although this was not captured objectively). Regardless of the heading technique adopted, both boys and girls in the intervention group reduced head impact magnitude (most notably peak linear acceleration for all players and peak angular velocity for predominantly girls) when compared with control players. Previous research [30, 36] has also shown that improved recruitment of neck muscles during heading resulted in decreased head acceleration on ball impact. A recommendation for future research is to analyse variations in heading technique between boys and girls from different age groups and experience levels as well as at different ball speeds using a much larger sample size of players. It is possible that the heading technique adopted by many girls in our study would result in different head impact magnitude data with higher velocity balls (replicating a kicked ball). In addition, accurately measuring head velocity as well as neck muscle activation, along with ball velocity prior to impact as well as the velocity and accuracy of the ball following a header would make important contributions to the literature [10].

\subsection{Limitations}

Players in both groups demonstrated improvements in isometric neck strength from baseline to follow-up, but the increase was much greater in players involved in the intervention group (53.8\%) when compared with the control $(15.6 \%)$. As the intervention period was only 5 weeks in duration, it is likely that some of this increase in both groups is attributable to players being more familiar and/or comfortable with the neck strength testing procedure. To account for this, we calculated the MDC to demonstrate the difference in change scores that could be attributed to a learning effect or measurement error. All outcome variables exceeded the MDC for the intervention group. It should also be noted that the mean baseline composite isometric neck strength of the players in the control arm $(15.9 \mathrm{~kg})$ was higher than the players in the intervention arm $(10.4 \mathrm{~kg})$, which was more pronounced in boys. This difference in strength is likely due to boys being older in the control arm (mean 15.3 years) when compared with the intervention arm (mean 13.8 years). It is likely that head impact magnitude during heading was influenced by these differences in age and strength for boys. Thus, it is plausible that the neck exercise programme used in our study provided greater benefit for younger players, but further research with a larger sample of age-matched players is required to elucidate this further. In addition, the result of our study in adolescent football players may not generalise to football players of different ages (i.e. young children or adult football players). It should also be noted that we only measured neck strength and not muscle activity, which would provide an important addition to a future study. When exploring sex differences, it should be noted that the 
a priori sample size calculation did not account for separate analyses by sex. The decision to review the results for all players as well as for boys and girls separately was made following data collection when the different heading technique adopted by the boys and girls in our study was noted. Based on our a priori sample size calculation $(n=46)$, our study was sufficiently powered for combined data $(n=52)$, but underpowered for boys' $(n=31)$ and girls' $(n=21)$ data when analysed separately, which should be acknowledged when interpreting our results by sex. It is recommended that this trial is repeated using a larger sample size of male and female players to further explore the influence of sex on head impact magnitude during heading.

Previous research has indicated that poor coupling of head mounted IMUs may lead to an overestimation of peak head impact magnitude during heading when compared with an instrumented mouthguard under laboratory conditions [48]. Pilot testing of our measures revealed no detectable slippage of the 'swim cap' during heading when compared with video analysis. An instrumented mouthguard was also not used, as football players do not routinely wear mouthguards, making it possible that just wearing a mouthguard could have influenced head impact magnitude through teeth clenching, which has been shown to reduce head impact magnitudes during heading and with blows to the head [49, 50]. However, it should be acknowledged that the methodology for measuring head kinematics using the head mounted IMU was not validated against a gold standard measure (such as using a National Operating Committee on Standards for Athletic Equipment [NOCSAE] headform model). Further, it is also possible that the longer hair of many girls may have reduced the coupling of the head mounted IMU, which may also have dampened the head impact magnitude of girls, which in part, may explain the lower head impact magnitudes of girls (although this would not have influenced the effect of the intervention as testing conditions remained the same at baseline and follow-up for all participants). Finally, it should be noted that the ball pressure used in our study (5 psi) was lower than the ball pressure recommended for match balls by the International Football Association Board (8.5-15.6 psi). While the generalisability of our results may be limited to lower ball pressures, the differences between the intervention and control groups indicate a positive effect of neck exercises for reducing head accelerations during purposeful heading.

\section{Conclusion}

Male and female adolescent players who completed neuromuscular neck exercises demonstrated an increase in isometric neck strength and a decrease in head impact magnitude during heading. Given these exercises took less than $2 \mathrm{~min}$ to complete and that they were perceived as feasible and acceptable by players and coaches involved in our study, the integration of neuromuscular neck exercises into part 2 of the FIFA $11+$ for adolescent players warrants further investigation. It is also recommended that a larger trial is conducted to explore the effects of this neuromuscular neck exercise programme on the incidence of head and neck injuries (including concussion) in various age groups, female and male players and players with different experience levels.

Supplementary Information The online version contains supplementary material available at https://doi.org/10.1007/s40279-021-01564-0.

Acknowledgements We would like to acknowledge David Mitchell for his unfaltering assistance with throw-ins and developing the game scenario as well as Edward Ferguson and Jason Eagar for their assistance with recruitment. Additionally, we wish to thank the coaches, players and parents for their involvement with this study.

\section{Declarations}

Funding This study was funded by a FIFA Research Scholarship grant awarded to Kerry Peek and Andrew Gardner.

Conflict of interest Kerry Peek, Jordan Andersen, Marnee McKay, Theo Versteegh, Ian Gilchrist and Tim Meyer declare that they have no conflicts of interest. Andrew Gardner, PhD. serves as a scientific advisor for hitIQ, Ltd. He has a clinical practice in neuropsychology involving individuals who have sustained sport-related concussion (including current and former athletes). He has been a contracted concussion consultant to Rugby Australia since July 2016. He has received travel funding or been reimbursed by professional sporting bodies and commercial organisations for discussing or presenting sport-related concussion research at meetings, scientific conferences, workshops and symposiums. Previous grant funding includes the NSW Sporting Injuries Committee, the Brain Foundation (Australia), an AustralianAmerican Fulbright Commission Postdoctoral Award, a Hunter New England Local Health District, Research, Innovation and Partnerships Health Research \& Translation Centre and Clinical Research Fellowship Scheme, and the Hunter Medical Research Institute (HMRI), supported by Jennie Thomas, and the HMRI, supported by Anne Greaves. $\mathrm{He}$ is currently funded through an NHMRC Early Career Fellowship and the University of Newcastle's Priority Research Centre for Stroke and Brain Injury. He has received research funding from the National Rugby League (NRL) for the Retired Players Brain Health research programme.

Availability of data and material The datasets generated during and/or analysed during the current study are available from the corresponding author on reasonable request.

Code availability The code used in the current study is available from Jordan Andersen via the corresponding author on reasonable request.

Authors' contributions Kerry Peek, Jordan Andersen, Marnee McKay, Tim Meyer and Andrew Gardner contributed to the concept and design of this study. Kerry Peek, Marnee McKay and Jordan Andersen pilot tested the IMU in the biomechanics laboratory prior to the study commencing. Kerry Peek and Theo Versteegh designed the exercise programme used in this study. Kerry Peek and Marnee McKay collected the data. Jordan Andersen converted and calculated head impact data. The first draft of the manuscript was written by Kerry Peek with initial 
review from Andrew Gardner. All authors commented on versions of the manuscript, and all read and approved the final manuscript.

Ethics approval Approval for the research was granted from the Human Research Ethics Committee at the University of Sydney. This study was performed in accordance with the standards of ethics outlined in the Declaration of Helsinki and registered with the Australian New Zealand Clinical Trials Registry (no: ACTRN12619001375145).

Consent to participate All participants and their parent/carer/guardian provided written consent to participate prior to study commencement.

Consent for publication All participants and their parent/carer/guardian provided written consent for publication of their data prior to study commencement

\section{References}

1. McCunn R, Beaudouin F, Stewart K, Meyer T, MacLean J. Heading in football: incidence, biomechanical characteristics and the association with acute cognitive function-a three-part systematic review. Sports Med. 2021;51:2147-63.

2. Russell ER, Mackay DF, Stewart K, MacLean JA, Pell JP, Stewart W. Association of field position and career length with risk of neurodegenerative disease in male former professional soccer players. JAMA Neurol. 2021;78(9):1057-63.

3. Tarnutzer AA, Straumann D, Brugger P, Feddermann-Demont N. Persistent effects of playing football and associated (subconcussive) head trauma on brain structure and function: a systematic review of the literature. Br J Sports Med. 2017;51(22):1592-604.

4. Wang F, Han Y, Wang B, Peng Q, Huang X, Miller K, et al. Prediction of brain deformations and risk of traumatic brain injury due to closed-head impact: quantitative analysis of the effects of boundary conditions and brain tissue constitutive model. Biomech Model Mechanobiol. 2018;17(4):1165-85.

5. Taha Z, Hassan MHA, Hasanuddin I. Analytical modelling of soccer heading. Sadhana. 2015;40(5):1567-78.

6. Kuo C, Wu L, Zhao W, Fanton M, Ji S, Camarillo DB. Propagation of errors from skull kinematic measurements to finite element tissue responses. Biomech Model Mechanobiol. 2018;17(1):235-47.

7. Cecchi NJ, Monroe DC, Moscoso WX, Hicks JW, Reinkensmeyer DJ. Effects of soccer ball inflation pressure and velocity on peak linear and rotational accelerations of ball-to-head impacts. Sports Eng. 2020;23(1):1-6.

8. Shewchenko N, Withnall C, Keown M, Gittens R, Dvorak J. Heading in football. Part 2: biomechanics of ball heading and head response. Br J Sports Med. 2005;39:i26-32.

9. Peek K, McKay M, Fu A, Meyer T, Oxenham V, Esopenko C, et al. The effect of ball characteristics on head acceleration during purposeful heading in male and female youth football players. Sci Med Footb. 2021. https://doi.org/10.1080/24733938.2021.18976 57.

10. Caccese JB, Kaminski TW. Minimizing head acceleration in soccer: a review of the literature. Sports Med. 2016;46(11):1591-604.

11. Peek K, Elliott JM, Orr R. Higher neck strength is associated with lower head acceleration during purposeful heading in soccer: a systematic review. J Sci Med Sport. 2020;23(5):453-62.

12. Spiotta AM, Bartsch AJ, Benzel EC. Heading in Soccer: dangerous play? Neurosurgery. 2012;70(1):1-11.

13. Putukian M, Echemendia RJ, Chiampas G, Dvorak J, Mandelbaum B, Lemak LJ, et al. Head Injury in Soccer: From Science to the Field; summary of the head injury summit held in April 2017 in New York City, New York. Br J Sports Med. 2019;53(21):1332-6.
14. Maher ME, Hutchison M, Cusimano M, Comper P, Schweizer TA. Concussions and heading in soccer: a review of the evidence of incidence, mechanisms, biomarkers and neurocognitive outcomes. Brain Inj. 2014;28(3):271-85. https://doi.org/10.3109/02699052. 2013.865269.

15. Yang YT, Baugh CM. US youth soccer concussion policy: heading in the right direction. JAMA Pediatr. 2016;170(5):413-4.

16. Football Association (England). Updated Heading Guidance. 2021. https://www.thefa.com/news/2021/jul/28/20210728-newheading-guidance-published Accessed 17 Aug 2021.

17. Peek K, Meyer T, Beaudouin F, McKay M. Heading incidence in boys' football over three seasons. Sci Med Footb. 2020;5(3):175-80.

18. Dick R, Putukian M, Agel J, Evans TA, Marshall SW. Descriptive epidemiology of collegiate women's soccer injuries: National Collegiate Athletic Association Injury Surveillance System, 19881989 through 2002-2003. J Athl Train. 2007;42(2):278.

19. Babbs CF. Biomechanics of heading a soccer ball: implications for player safety. Sci World J. 2001;1:281-322.

20. Koerte IK, Schultz V, Sydnor VJ, Howell DR, Guenette JP, Dennis E, et al. Sex-related differences in the effects of sports-related concussion: a review. J Neuroimaging. 2020;30(4):387-409.

21. Pfister T, Pfister K, Hagel B, Ghali WA, Ronksley PE. The incidence of concussion in youth sports: a systematic review and meta-analysis. Br J Sports Med. 2016;50(5):292-7.

22. Tierney RT, Higgins M, Caswell SV, Brady J, McHardy K, Driban JB, et al. Sex differences in head acceleration during heading while wearing soccer headgear. J Athl Train. 2008;43(6):578-84.

23. Caccese JB, Buckley TA, Tierney RT, Rose WC, Glutting JJ, Kaminski TW. Sex and age differences in head acceleration during purposeful soccer heading. Res Sports Med. 2018;26(1):64-74. https://doi.org/10.1080/15438627.2017.1393756.

24. Tierney RT, Sitler MR, Swanik CB, Swanik KA, Higgins M, Torg J. Gender differences in head-neck segment dynamic stabilization during head acceleration. Med Sci Sports Exerc. 2005;37(2):272-9.

25. Elliott J, Heron N, Versteegh T, Gilchrist IA, Webb M, Archbold $\mathrm{P}$, et al. injury reduction programs for reducing the incidence of sport-related head and neck injuries including concussion: a systematic review. Sports Med. 2021. https://doi.org/10.1007/ s40279-021-01501-1.

26. Attwood MJ, Roberts SP, Trewartha G, England ME, Stokes KA. Efficacy of a movement control injury prevention programme in adult men's community rugby union: a cluster randomised controlled trial. Br J Sports Med. 2018;52(6):368-74.

27. Hislop MD, Stokes KA, Williams S, McKay CD, England ME, Kemp SPT, et al. Reducing musculoskeletal injury and concussion risk in schoolboy rugby players with a pre-activity movement control exercise programme: a cluster randomised controlled trial. Br J Sports Med. 2017;51(15):1140-6.

28. Bizzini M, Dvorak J. FIFA 11+: an effective programme to prevent football injuries in various player groups worldwide - a narrative review. Br J Sports Med. 2015;49(9):577-9.

29. Caccese JB, Buckley TA, Tierney RT, Arbogast KB, Rose WC, Glutting JJ, et al. Head and neck size and neck strength predict linear and rotational acceleration during purposeful soccer heading. Sports Biomech. 2018;17(4):462-76.

30. Müller $\mathrm{C}$, Zentgraf $\mathrm{K}$. Neck and trunk strength training to mitigate head acceleration in youth soccer players. J Strength Cond Res. 2020. https://doi.org/10.1519/jsc.0000000000003822.

31. Schulz KF, Altman DG, Moher D, the CG. CONSORT 2010 Statement: updated guidelines for reporting parallel group randomised trials. BMC Med. 2010;8(1):18. https://doi.org/10.1186/ 1741-7015-8-18.

32. Thorborg K, Krommes KK, Esteve E, Clausen MB, Bartels EM, Rathleff MS. Effect of specific exercise-based football injury 
prevention programmes on the overall injury rate in football: a systematic review and meta-analysis of the FIFA 11 and 11+ programmes. Br J Sports Med. 2017;51(7):562-71.

33. Hamilton D, Gatherer D, Jenkins P, Maclean J, Hutchison J, Nutton R, et al. Age-related differences in the neck strength of adolescent rugby players: a cross-sectional cohort study of Scottish schoolchildren. Bone Jt Res. 2012;1(7):152-7.

34. Hamilton DF, Gatherer D. Cervical isometric strength and range of motion of elite rugby union players: a cohort study. BMC Sports Sci Med Rehabil. 2014;6(1):32.

35. Peek K, Gatherer D. The rehabilitation of a professional Rugby Union player following a C7/T1 posterior microdiscectomy. Phys Ther Sport. 2005;6(4):195-200.

36. Worsey MT, Jones BS, Cervantes A, Chauvet SP, Thiel DV, Espinosa HG. Assessment of head impacts and muscle activity in soccer using a $\mathrm{t} 3$ inertial sensor and a portable electromyography (emg) system: a preliminary study. Electronics. 2020;9(5):834.

37. Peek K, Vella T, Meyer T, Beaudouin F, McKay M. The incidence and characteristics of purposeful heading in male and female youth football (soccer) within Australia. J Sci Med Sport. 2021;24(6):603-8.

38. Koo TK, Li MY. A guideline of selecting and reporting intraclass correlation coefficients for reliability research. J Chiropr Med. 2016;15(2):155-63.

39. Garces GL, Medina D, Milutinovic L, Garavote P, Guerado E. Normative database of isometric cervical strength in a healthy population. Med Sci Sports Exerc. 2002;34(3):464-70.

40. Dontje ML, Dall PM, Skelton DA, Gill JMR, Chastin SFM, Seniors USPT. Reliability, minimal detectable change and responsiveness to change: Indicators to select the best method to measure sedentary behaviour in older adults in different study designs. PLoS One. 2018;13(4):e0195424-e. https://doi.org/10.1371/journ al.pone.0195424.

41. Gupta SK. Intention-to-treat concept: a review. Perspect Clin Res. 2011;2(3):109.
42. Naish R, Burnett A, Burrows S, Andrews W, Appleby B. Can a Specific neck strengthening program decrease cervical spine injuries in a men's professional rugby union team? A retrospective analysis. J Sports Sci Med. 2013;12(3):542-50.

43. Ylinen JJ, Rezasoltani A, Julin MV, Virtapohja HA, Mälkiä EA. Reproducibility of isometric strength: measurement of neck muscles. Clin Biomech. 1999;14(3):217-9.

44. Becker S, Berger J, Backfisch M, Ludwig O, Kelm J, Fröhlich M. Effects of a 6-week strength training of the neck flexors and extensors on the head acceleration during headers in soccer. J Sports Sci Med. 2019;18(4):729.

45. Shewchenko N, Withnall C, Keown M, Gittens R, Dvorak J. Heading in football. Part 1: development of biomechanical methods to investigate head response. Br J Sports Med. 2005;39:110-25.

46. Hildenbrand KJ, Vasavada AN. Collegiate and high school athlete neck strength in neutral and rotated postures. J Strength Cond Res. 2013;27(11):3173-82. https://doi.org/10.1519/JSC.0b013e3182 $8 \mathrm{a} 1 \mathrm{fe} 2$.

47. Alsalaheen B, Johns K, Bean R, Almeida A, Eckner J, Lorincz $M$. Women and men use different strategies to stabilize the head in response to impulsive loads: implications for concussion injury risk. J Orthop Sports Phys Ther. 2019;49(11):779-86.

48. Wu LC, Nangia V, Bui K, Hammoor B, Kurt M, Hernandez F, et al. In vivo evaluation of wearable head impact sensors. Ann Biomed Eng. 2016;44(4):1234-45. https://doi.org/10.1007/ s10439-015-1423-3.

49. Tanaka Y, Tsugawa T, Maeda Y. Effect of mouthguards on impact to the craniomandibular complex. Dent Traumatol. 2017;33(1):51-6.

50. Keishiro N, Tomotaka T, Kazunori N, Michiyo K, Takamitsu O, Keiichi I. Effect of clenching with a mouthguard on head acceleration during heading of a soccer ball. Gen Dent. 2015;63(6):41-7.

\section{Authors and Affiliations}

\section{Kerry Peek ${ }^{1}$ (I) Jordan Andersen ${ }^{2} \cdot$ Marnee J. McKay $^{1} \cdot$ Theo Versteegh $^{3} \cdot \operatorname{lan}$ A. Gilchrist ${ }^{4} \cdot$ Tim Meyer $^{5}$. Andrew Gardner ${ }^{6,7}$ (B)}

Jordan Andersen

jordan.andersen@mq.edu.au

Marnee J. McKay

marnee.mckay@sydney.edu.au

Theo Versteegh

tverstee@uwo.ca

Ian A. Gilchrist

ig13@queensu.ca

Tim Meyer

tim.meyer@mx.uni-saarland.de

Andrew Gardner

Andrew.Gardner@newcastle.edu.au

1 Discipline of Physiotherapy, School of Health Sciences, Faculty of Medicine and Health, The University of Sydney, Susan Wakil Health Building, Camperdown, Sydney, NSW, Australia
2 School of Health Sciences, Faculty of Medicine and Health, The University of Sydney, Sydney, NSW, Australia

3 School of Physical Therapy, Western University, London, ON, Canada

4 School of Kinesiology and Health Studies, Queen's University, Kingston, ON, Canada

5 Institute of Sports and Preventive Medicine, Medical Faculty, Saarland University, Saarbrücken, Germany

6 Priority Research Centre for Stroke and Brain Injury, School of Medicine and Public Health, The University of Newcastle, Newcastle, NSW, Australia

7 Hunter New England Local Health District Sports Concussion Research Program, Calvary Mater Hospital, Waratah, NSW, Australia 